\title{
Mechanical Properties of Carbon Fiber-Reinforced Aluminum Alloys with Whiskers and Particulates of Silicon-Carbide
}

\author{
By Shin-ichi Towata*, Hajime Ikuno* and Sen-ichi Yamada*
}

\begin{abstract}
New types of carbon fiber-reinforced aluminum alloys, in which whiskers and fine particulates of silicon-carbide were distributed among the fibers, were prepared by a squeeze casting process, using a low cost high strength type carbon fibers. The mechanical properties and fracture morphologies of the composites were investigated by the three-point flexural tests with acoustic emission measurements. The results obtained are as follows.

In the conventional composites, the longitudinal flexural strength was very low and the failure of the fibers initiated mainly at the point of direct fiber-to-fiber contacts. On the other hand, the longitudinal flexural strength of the hybrid composite was about $50 \%$ higher than that of the conventional composites. In the hybrid composites, the fibers were separated by whiskers and particulates. The failure occurred after accumulation of microcracks of fiber break.
\end{abstract}

(Received October 2, 1987)

Keywords: carbon fiber, whisker, particulate, hybrid composite, mechanical properties, fracture morphology

\section{Introduction}

Fiber-reinforced metal matrix composites are recently expected to be a candidate of structural materials in automobile and aerospace industries $^{(1)}$. As reinforcements, many kinds of fibers have been developed such as carbon, silicon-carbide, boron and alumina fibers. The use of carbon fibers, particularly, allows the weight reduction because of their high modulus-to-weight and strength-to-weight ratios. Aluminum alloys are used as the metal matrix because of their good handling ability. Many studies have been performed for the development of high performance carbon fiber-reinforced aluminum alloys ${ }^{(2)-(8)}$. Molten aluminum alloys do not wet carbon fibers. Also, brittle reaction products of aluminumcarbide which cause the degradation of the strength of carbon fiber are formed at a high temperature ${ }^{(8)}$. Therefore, the surface treatments and coatings are required for increasing the wettability and suppressing the interfacial

* Toyota Central Research \& Development Laboratories, Inc., Aichi 480-11, Japan. reactions. High modulus type carbon fibers are usually expected as a reinforcement of aluminum alloys because of their lower reactivity, but they are expensive.

Squeeze casting or pressure casting is interesting as an industrial fabrication process of metal matrix composites. Silicon-carbide fiber $^{(9)}$, alumina fiber ${ }^{(10)}$ and alumina-silica fiber ${ }^{(11)}$ have been successfully incorporated into aluminum alloys by a squeeze casting process. High modulus type carbon fiber-reinforced aluminum alloys, which have the strength corresponding to that predicted by the rule-of-mixture, have been fabricated even by the squeeze casting process with no surface treatment $^{(12)}$.

In the present study, high strength type carbon fibers, which are at low cost and have not been successfully used as the reinforcement of aluminum alloys, were incorporated into aluminum alloys by the squeeze casting process with the addition of fine particulates and whiskers and without surface treatment of fibers. The effect of hybrid technique (additions of fine particulates and whiskers) on the mechanical properties and fracture behavior of the composites were investigated. 
Table 1 Properties of reinforcements.

\begin{tabular}{ll}
\hline \hline Carbon Fiber*1 $^{* 1}$ & Dia. $=7 \mu \mathrm{m}$ \\
& $E_{\mathrm{f}}=240 \mathrm{GPa}$ \\
\hline Whisker $^{* 2}$ & Silicon-carbide \\
& Dia. $=0.2 \mu \mathrm{m}$ \\
& Length $=40 \mu \mathrm{m}$ \\
\hline Particulate $^{* 3}$ & Silicon-carbide \\
& Dia. $=0.28 \mu \mathrm{m}$ \\
\hline
\end{tabular}

*1 Toho Rayon Co., Ltd.

*2 Tokai Carbon Co., Ltd.

${ }^{* 3}$ Ibiden Co., Ltd.

\section{Experimental Procedure}

The carbon fibers employed in the present study were high strength type PAN based fibers (Toho Rayon "ST-II-7"). The additional reinforcements were whiskers and particulates of silicon-carbide. The details of the reinforcements are listed in Table 1. Figure 1 shows the size distribution of silicon-carbide particulates. The whiskers and particulates were uniformly incorporated into continuous carbon fiber bundles in advance ${ }^{(13)}$. Figure 2 shows the hybrid carbon fibers with whiskers and particulates. The hybrid fiber bundles were cut to the length of $150 \mathrm{~mm}$ and were shaped into a preform plate about $2 \mathrm{~mm}$ thick. The squeeze casting process ${ }^{(12)}$ was carried out at a pressure of $90 \mathrm{MPa}$ and a pressing time of $60 \mathrm{~s}$. The matrices were pure aluminum and $\mathrm{Al}-5 \% \mathrm{Mg}$ alloy. Also the conventional composites reinforced with carbon fibers were fabricated under the same casting conditions. The volume fraction of fibers and additional reinforcements for the hybrid composites were 0.52 and 0.07 , respectively, against the respective values of 0.7 and zero for the conventional ones.

The cross-sections of the composites were polished with silicon-carbide emery paper and diamond paste (diameter: 1 micron) and were subjected to analysis with a scanning electron microscope.

The longitudinal mechanical properties were investigated under a flexural mode. Threepoint flexural tests were carried out by use of the coupons of $100 \mathrm{~mm} \times 8 \mathrm{~mm} \times 1.8 \mathrm{~mm}$,

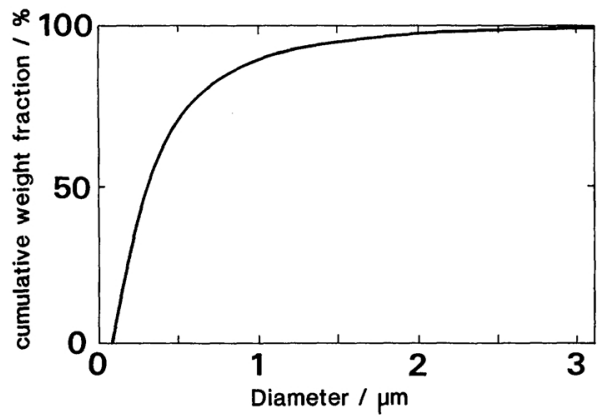

Fig. 1 Size distribution of silicon-carbide particulates.

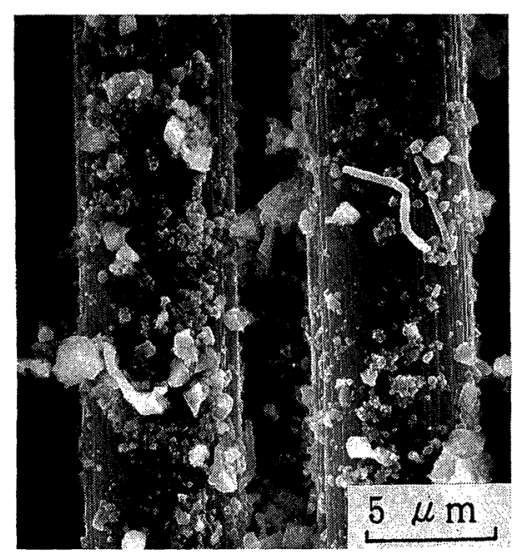

Fig. 2 Scanning electron micrographs of carbon fibers with whiskers and particulates.

which were mechanically polished with emery paper of about 1000 grits before tests. Span was $90 \mathrm{~mm}$ and crosshead speed was 0.033 $\mathrm{mm} / \mathrm{s}$. In addition, acoustic emission (AE) signals were monitored during the flexural tests using the Dunegan/Endevco 3000 AE system. The threshold level was $25 \mathrm{~dB}$. The details of $\mathrm{AE}$ measurements have been described elsewhere $^{(14)}$.

The transverse flexural properties were examined using the coupons of $18 \mathrm{~mm} \times 10$ $\mathrm{mm} \times 1.8 \mathrm{~mm}$. In the three-point flexural tests, the span was $15 \mathrm{~mm}$ and the crosshead speed was $0.017 \mathrm{~mm} / \mathrm{s}$.

In addition to the mechanical properties of the composites, the tensile strength of carbon fibers was measured using a miniature tensile test machine. The fibers were extracted from the composites in a warm $10 \%$ sodium hydrooxide aqueous solutions and were dried in air 

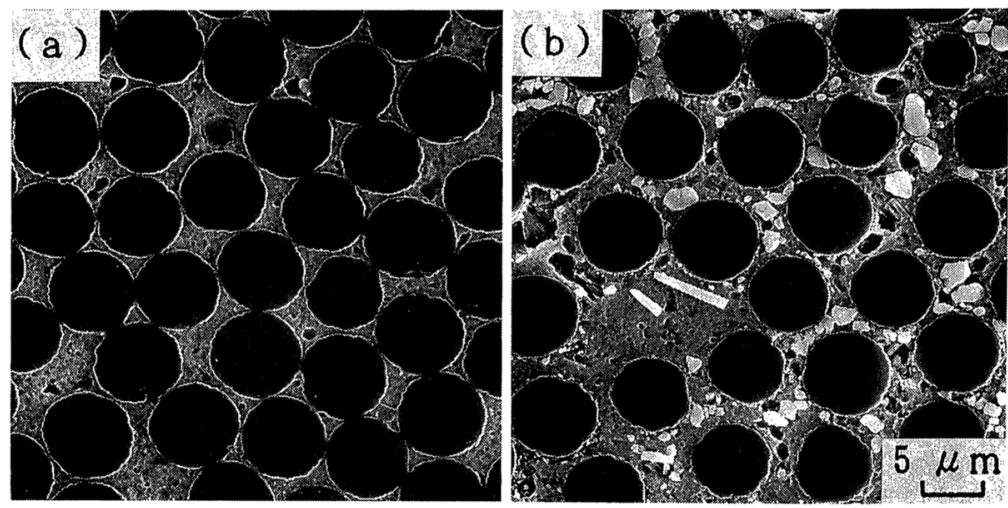

Fig. 3 Scanning electron micrographs of cross sections of composite. (a) conventional composite, (b) hybrid composite.

at room temperature. The diameters of all the fibers (about 40 filaments for each sample) were measured by the method of diffraction pattern analysis of the $\mathrm{He}-\mathrm{Ne}$ gas laser beam (0.8 $\mathrm{mm}$ diameter).

\section{Experimental Results}

\section{Microstructures of composites}

The scanning electron micrographs of crosssections of composites are shown in Fig. 3. Aluminum matrix was sufficiently infiltrated into cavities among the fibers in both of the conventional and hybrid composites. Whereas, the two types of composites exhibited distinctly different morphological features, particularly the fiber distribution. Nearly all the fibers are in direct contact with adjacent fibers and some of them are close-packed in the conventional composite. On the other hand, the fibers are separated with sufficient fiber spacing in the hybrid composite. Whiskers with random orientations and particulates, which are shown in the figure with bright contrast image, are distributed among the fibers. Interfacial reaction products, such as aluminum carbide were not observed on the fiber surfaces by scanning electron microscopy.

\section{Longitudinal flexural properties}

The longitudinal flexural strength is illustrated in Fig. 4. While the volume fraction of carbon fiber was about $25 \%$ higher in the conventional composites than in the hybrid ones, the flexural strength was about 50\% higher in the hybrid composites. The strength of the aluminum-magnesium alloy matrix composite was slightly higher than the pure aluminum matrix ones. The flexural modulus
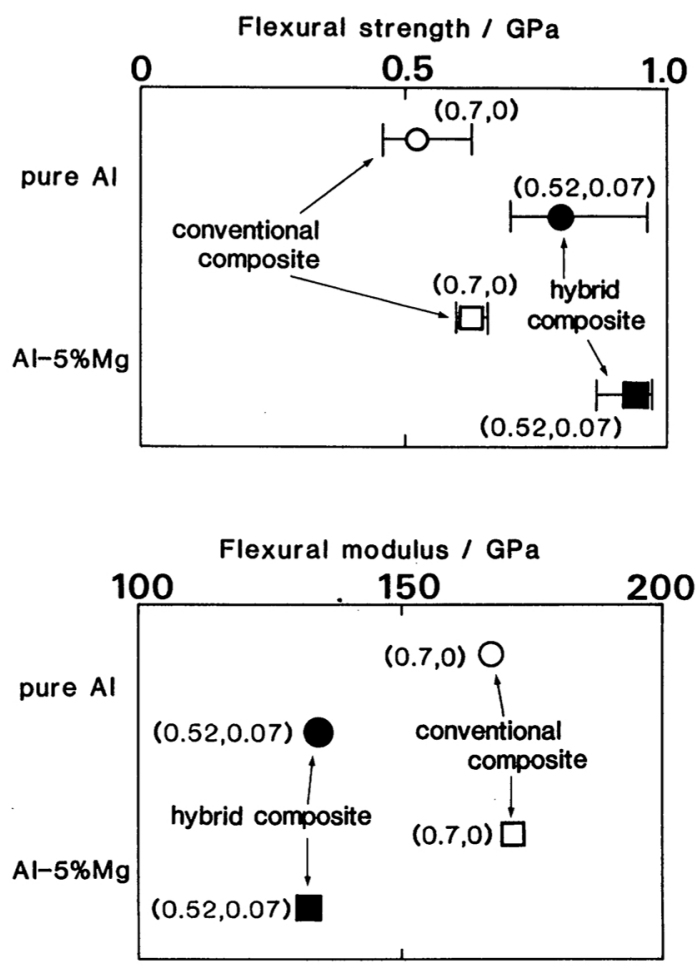

Fig. 4 Longitudinal flexural properties of composites. The numbers in the parentheses are represent the volume fraction of fibers and (particulates + whiskers). 


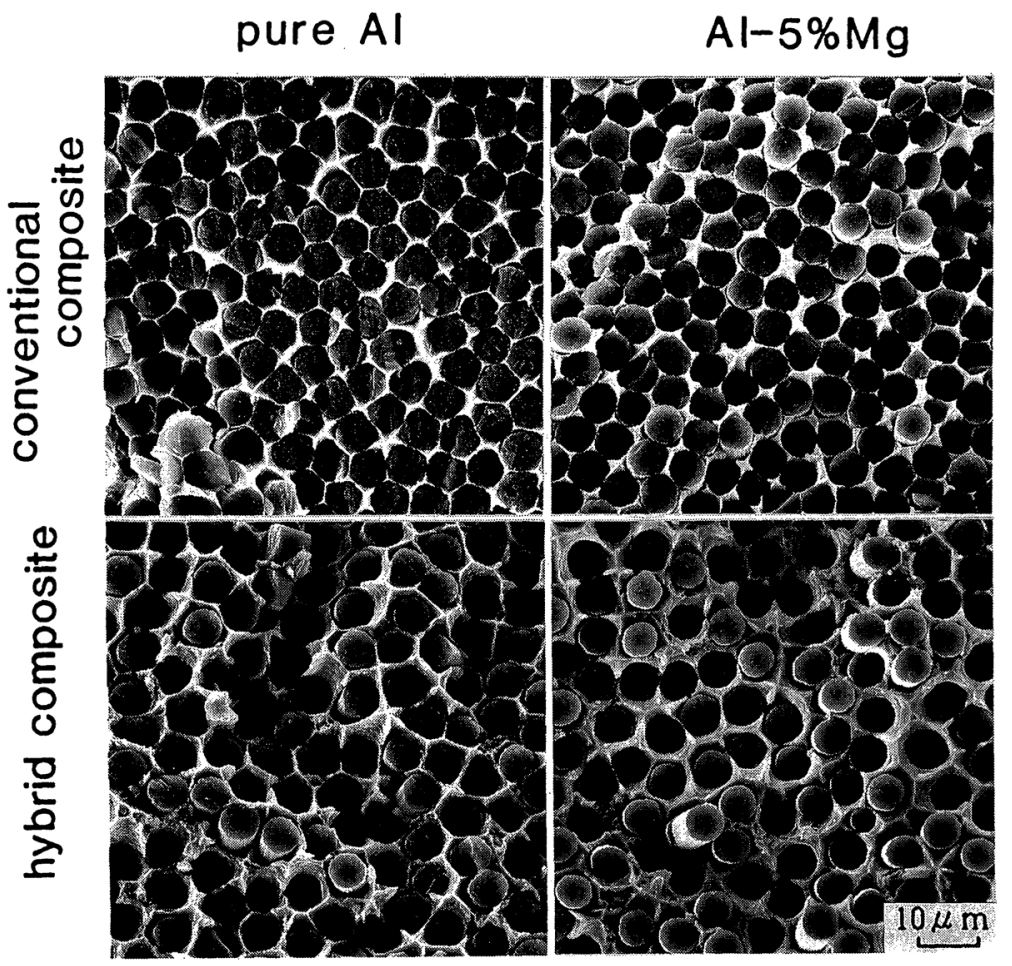

Fig. 5 Scanning electron micrographs of longitudinal flexural fracture surfaces of composites.

was lower in the hybrid composites, which is reasonable in consideration of the volume fraction of carbon fibers.

The typical fracture surfaces are shown in Fig. 5. The conventional composites exhibited flatter surfaces than the hybrid ones and the fracture of the fibers was initiated at the direct fiber-to-fiber contact points. The fracture of one fiber was related to the successive fracture of many other adjacent fibers. The direction of the fracture propagation of fibers was not so clear for the hybrid composites, because the fibers were separated with substantial fiber spacings. It was to be noticed in the hybrid composites that the fibers were occasionally pulled out and the number and length of pulled-out fibers was slightly larger in the aluminum-magnesium matrix composite. Small dimple patterns were frequently observed in the matrix regions. These patterns are caused by the addition of whiskers and particulates.

The different fracture behavior of the two types of composites will be further revealed by
AE measurements. The events and ringdown versus deflection curves in AE measurements during longitudinal three-point flexural tests are illustrated in Fig. 6.

For the conventional composites, the flexural stress increased linearly with the deflection until a drastic fracture. AE ringdown counts rapidly increased just before the fracture. The abrupt increase of ringdown counts is caused by a openning of larger cracks. The total events and ringdown counts from the start of loading to fracture was much larger in the aluminum-magnesium matrix composite.

On the other hand, for the hybrid composites, both of the events and ringdown counts increased gradually until the occurence of fracture. This behavior corresponds to a progressive anelastic deformation, which is presumable from the stress-deflection curves.

From the AE measurements and fractographs, the fracture mechanisms of these two types of composites can be considered as follows.

For the conventional composites, the small 

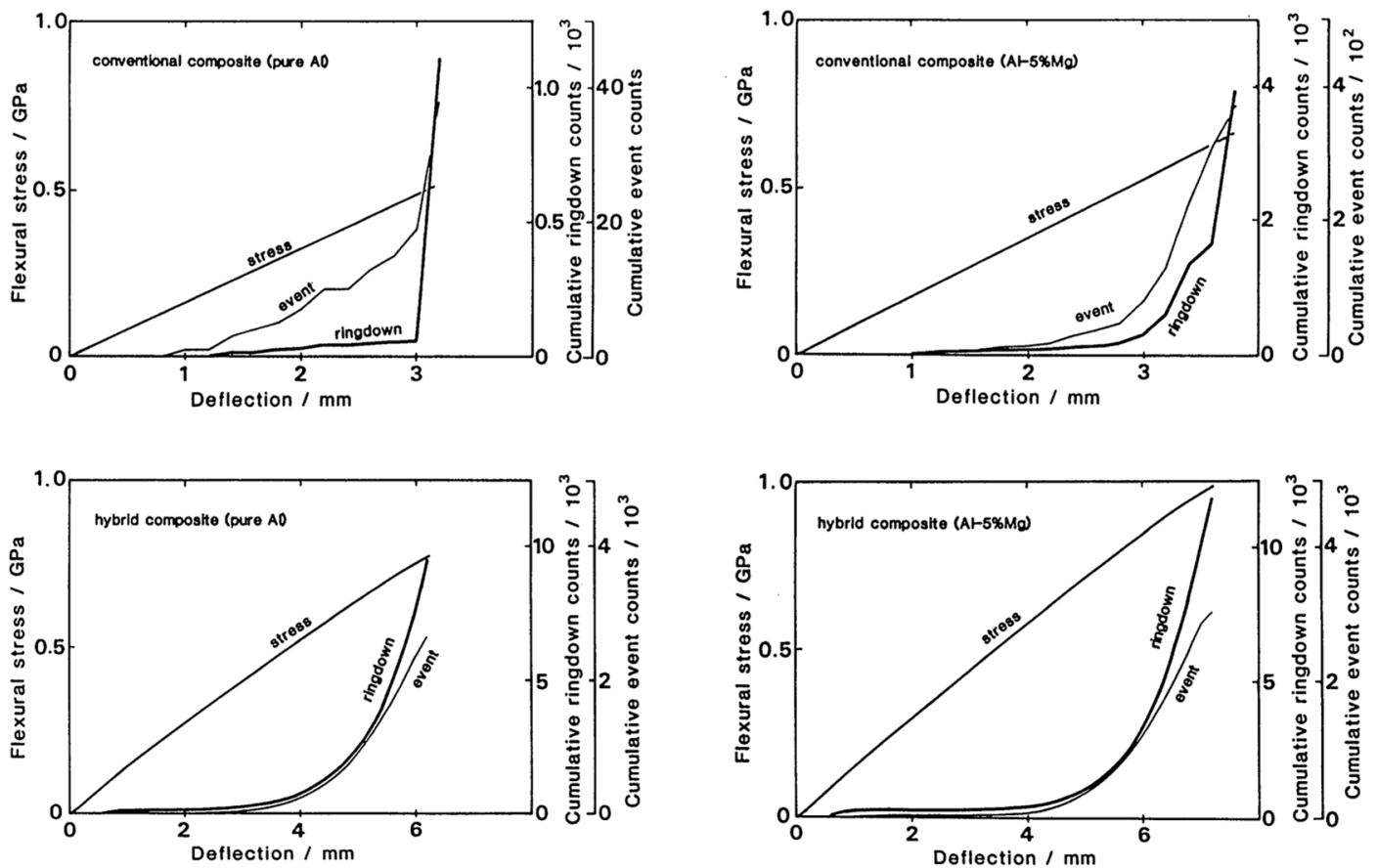

Fig. 6 Acoustic emission characteristics of composite during longitudinal flexural tests.

cracks caused by the fracture of weak fibers propagate rapidly to adjacent fibers through the direct fiber-to-fiber contact points, resulting in a drastic failure. This failure mode is classified as "non-accumulative failure".

For the hybrid composites, the small cracks of fiber break are formed accumulatively with the increase of stress. These cracks are propagate to the nearest cracks through the matrix at higher stress than about $0.5 \mathrm{GPa}$, followed by the rupture of the specimen.

\section{Transverse flexural properties}

Transverse properties are very important for the anisotropic materials such as unidirectionally fiber-reinforced metal matrix composites. The transverse mechanical properties were investigated in a three-point flexural mode. The results are illustrated in Fig. 7. For pure aluminum matrix composites, the flexural strength is similar in both types of composites. Whereas the strength of the hybrid composites is higher than that of the conventional ones for the aluminum-magnesium matrix. Figure 8 shows typical transverse fracture surfaces. For the conventional composites, many fibers were longitudinally splitted in the pure aluminum matrix, while some were splitted and others exhibited the morphological feature similar to the bare fiber surfaces in aluminummagnesium matrix. In contrast, fiber surfaces were frequently observed in both of the hybrid composites. Moreover, a lot of small dimple patterns were observed in the matrix regions, which are attributable to the addition of whiskers and particulates.

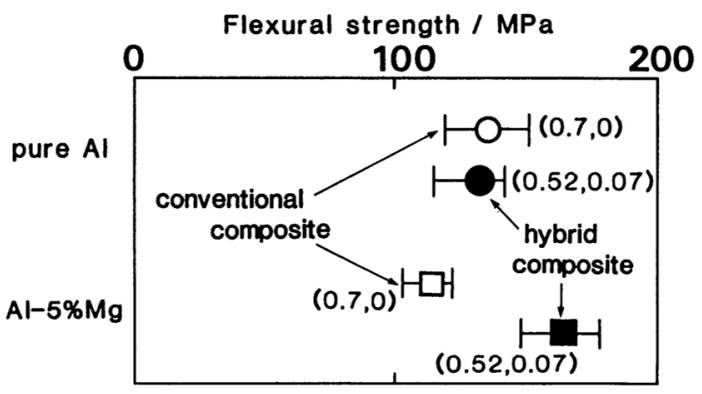

Fig. 7 Transverse flexural properties of composites. The numbers in the parentheses represent the volume fraction of fibers and (particulates + whiskers). 


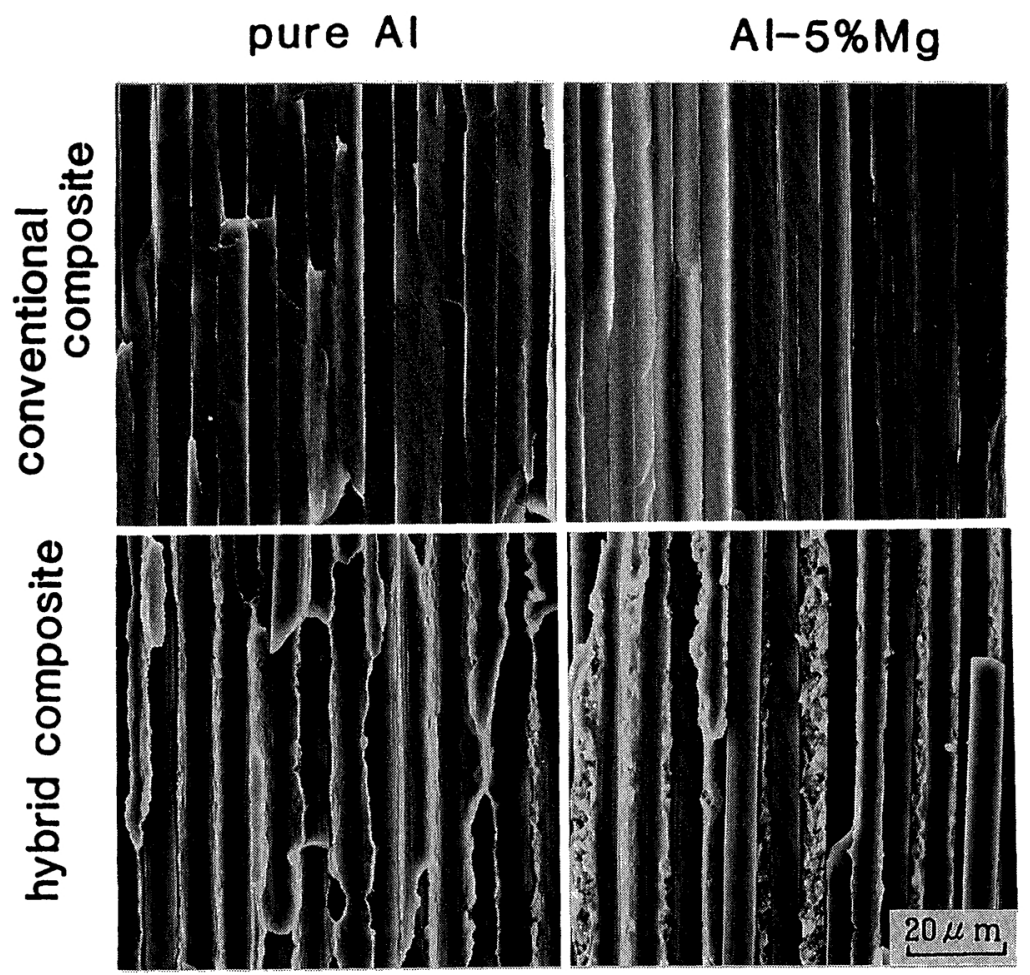

Fig. 8 Scanning electron micrographs of transverse flexural fracture surfaces of composites.

\section{Discussions}

The high strength type carbon fibers have been reported to be unsuitable as the reinforcements of aluminum alloys. Because the fibers are easy to be degraded by the interfacial reaction, when they are incorporated into aluminum alloys by the liquid infiltration process $^{(15)}$. In the present study, the conventional composites, which were reinforced with only continuous fibers, gave certainly low strength in the longitudinal direction. However, the hybrid composites indicated the strength of about $1.0 \mathrm{GPa}$, which was about $50 \%$ higher than those of the conventional composites, whereas both types of composites were fabricated in the same condition of squeeze casting.

The fiber strength is one of the factors controlling the strength of composites. Therefore, the fibers extracted from the composites were subjected to tensile tests. Table 2 shows the average fiber strength. It was found that the fiber degradation was less in the conventional composites. The fiber strength of aluminummagnesium matrix composite is similar to that of as-received fibers, nevertheless the fibers were incorporated into aluminum alloys at a higher temperature than the melting point of the matrix. On the other hand, the degradation of the fibers extracted from aluminum-

Table 2 Tensile strength of carbon fibers.

\begin{tabular}{llc}
\hline & & $\sigma_{\mathrm{f}}(\mathrm{GPa})$ \\
\hline As-received & 3.45 \\
\hline Pure Al & $\begin{array}{l}\text { Conventional } \\
\text { composite } \\
\text { Hybrid } \\
\text { composite }\end{array}$ & 2.79 \\
\hline $\mathrm{Al}-5 \% \mathrm{Mg}$ & $\begin{array}{l}\text { Conventional } \\
\text { composite } \\
\text { Hybrid } \\
\text { composite }\end{array}$ & 2.12 \\
\hline \hline
\end{tabular}

$\sigma_{\mathrm{f}}:$ Tensile strength of fiber 
magnesium matrix is similar to that of pure aluminum matrix in the hybrid composites. The fiber strength of the hybrid composites was lower than that of the conventional ones. The degradation of fibers in the hybrid composites may be caused by mechanical damage during the pre-treatment of adhering of whiskers and particulates onto fibers. It is suggested from these results that the strength of composites is not so attributable to the fiber strength as usually reported, but to the fracture mechanism ${ }^{(16)}$.

It have been found in the present study that the degradation of carbon fibers is influenced by the alloying elements in the matrix. Magnesium is not a strong carbide forming element like aluminum. In addition, the alloying elements of the matrix are segregated around the fibers in the liquid infiltration process ${ }^{(17)(18)}$. It is considered that magnesium concentrated around the fibers restricts the interfacial reaction between carbon fiber and aluminum. But, the effect of the alloying element of magnesium is not very clear. The details of this problem are now under study.

The authors reported that the direct fiber-tofiber contacts caused a heavy degradation of strength of silicon-carbide fiber-reinforced aluminum fabricated by a squeeze casting process, in terms of the crack propagation from broken fibers to adjacent fibers ${ }^{(16)}$. In the present study, the failure of many fibers was initiated at the direct contact points of adjacent fibers in the conventional composites. In contrast with this feature, the direction of propagation of fiber failure between the neighboring fibers was not very clear in the hybrid composites. The substantial improvement in the strength of composites can be accomplished by the hybrid technique mentioned above. The uniform distribution of fibers in the matrix without direct fiber-to-fiber contacts, which is one of the hybrid effects, will enable molten aluminum to completely infiltrate into fiber bundles and to suppress the stress concentration caused by the failure of the adjacent fibers.

The transverse fracture behavior involved two types of the modes, fiber splitting and interfacial failure. The former mode was observed in the conventional composites, particularly, in the pure aluminum matrix composite. The fiber splitting may be due to the anisotropic crystal structure of the fiber. Not simply the fiber degradation but the direct fiber contact causes the fiber splitting, because there were few splittings in the hybrid composites which indicated a lower fiber strength than the conventional composites. The hybrid composites exhibited mainly the interfacial failure mode. It is found that the interfacial bonding strength is lower than that of the matrix for the hybrid composites.

The transverse strength of composites is influenced by the strength of the matrix. On the other hand, for the hybrid composites most of the fibers are separated with a given inter-fiber spacing and the transverse failure occurs in the same mode. Therefore, the matrix will contribute effectively to the transverse strength of the composites.

The size of particulates or whiskers in the matrix is also an important factor affecting the mechanical properties of the composites. When the particulates among the carbon fibers are as large as the fiber, the crack propagation between fiber and particulate may have some influence on the mechanical properties of the composites, like fiber-to-fiber contacts. Some large particulates (diameter: about $2 \mu \mathrm{m}$ ) are observed in the transverse section (Fig. 3). The total amount of large particulates is small as shown in Fig. 1. Therefore, in the present study, the influence of them on the mechanical properties of the composites is negligible. However, the detail of the size effect of particulates needs further investigations.

\section{Conclusions}

New types of carbon fiber-reinforced aluminum alloys, in which whiskers and particulates were distributed among the fibers, were prepared by squeeze casting process, using low-cost high strength type carbon fibers. The mechanical properties and fracture morphologies of the composites were investigated.

(1) Longitudinal flexural strength of the composites increased by about $50 \%$ to $1.0 \mathrm{GPa}$ with the addition of silicon-carbide whiskers 
and particulates. The fiber failure was mainly initiated at the direct fiber-to-fiber contact points, resulting in the drastic failure for the conventional fiber composites. Most of the fibers were separated with an given inter-fiber spacing in the hybrid composites. The failure of hybrid composites was caused by the accumulation of micro-cracks.

(2) Transverse flexural strength was also improved by $50 \%$ by the hybrid technique.

(3) The degradation of carbon fibers was less in the conventional composites. But the strength of fibers extracted from the hybrid composites was lower than that of the conventional composites in contrast to higher longitudinal strength of the hybrid composites.

\section{Acknowledgment}

The authors would like to express their sincere thanks to Dr. Yamamura, Mr. Shibuya and Mr. Ishikawa of Ube Industries, Inc. for their useful discussions.

\section{REFERENCES}

(1) D. L. McDanels, T. T. Serafini and J. A. DiCarlo: J. Materials for Energy Systems, 8 (1986), 80.

(2) B. Blankenburgs: J. Austr. Inst. Met., 14 (1969), 236.

(3) A. A. Baker, Mrs. C. Shipman and P. W. Jakson:
Fibre Sci. and Tech., 5 (1972), 213.

(4) R. P. Pepper, J. W. Upp, R. C. Rossi and E. G. Kendall: Met. Trans., 2 (1971), 117.

(5) I. H. Kahn: Met. Trans. A, 7A (1976),1281.

(6) J. J. Tribendis, M. J. Koczak and R. Gracia: Powder Met. Internat., 13 (1981), 88.

(7) Y. Sawada and M. G. Bader: Proc. 5th Internat. Conf. on Composite Materials, ICCM-V, (1985), p. 785.

(8) S. J. Baker and W. Bonfield: J. Mater. Sci., 13 (1978), 1329.

(9) H. Fukunaga and T. Ohde: Proc. 4th Internat. Conf. on Composite Materials, ICCM-IV, (1982), p. 1443.

(10) Y. Abe, S. Horikiri, K. Fujimura and E. Ichiki: Proc. 4th Internat. Conf. on Composite Materials, ICCMIV, (1982), p. 1427.

(11) T. W. Clyne, M. G. Bader, G. R. Cappleman and P. A. Hubert: J. Mater. Sci., 20 (1985), 85.

(12) S. Towata and S. Yamada: Trans. JIM, 26 (1985), 563.

(13) S. Towata and S. Yamada: Proc. Japan-US Conference on Composite Materials, CCM-III, (1986), p. 497.

(14) S. Towata and S. Yamada: J. Japan Inst. Metals, 50 (1986), 336.

(15) M. F. Amateau: J. Composite Materials, 10 (1976), 279.

(16) S. Towata and S. Yamada: Trans. JIM, 27 (1986), 709.

(17) J. A. Cornie, A. Mortensen, M. N. Gunger and M. C. Flemings: Proc. 5th Internat. Conf. on Composite Materials, ICCM-V, (1985), p. 809.

(18) S. Towata, H. Ikuno and S. Yamada: J. Japan Inst. Metals, 51 (1987), 248. 